\title{
Research On The Evaluation Of Workshop Lean Level Based On ANP Xiaobing $\mathrm{Pei}^{1}$, Linlin $\mathrm{Jia}^{2, \mathrm{a}}$ \\ 1,2Tianjin University of Technology, Tianjin 300384, China \\ a13752157389@163.com
}

Keywords: Enterprise workshop, lean level, ANP, index weight, lean improvement.

\begin{abstract}
Based on the research background of enterprise workshop, the evaluation index system is set up, Analytic Network Process(ANP) is used, Decision Super computer software to determine the weight of indicators for the lean workshop level made a qualitative and quantitative research, which leads the enterprise to carry out the lean improvement, not only can the difference in each workshop, but also to the benchmarking enterprise as the standard, with direction of continuous improvement, to enhance their ability to survive and develop.
\end{abstract}

\section{Introduction}

Today the face of the market demand for small quantity and variety, lean production is the best choice for the survival and development of enterprises, and the level of lean is an important criterion of the lean production in the enterprise application. First of all, from the horizontal perspective, the enterprise can understand all of the lean workshop level, respectively, to find out in a lean low level within each workshop, there are differences in adjusting and improving, balance and improve the level of the lean enterprise workshop, to achieve the stability of the production system, strengthening the viability of the enterprise; Second, from longitudinal perspective, can help enterprises to realize clearly the lean level and benchmarking the gap of the enterprise, which can have a direction to continue to improve, enhance the market competitiveness of enterprises, realize the continuous development of enterprises.

\section{Literature review}

In recent years, many scholars lean enterprise level for a certain amount of research. Abroad, Sanjay Bhasin found that Lean Production Balanced Scorecard method adopted to reflect the impact of the enterprise is not enough, so the proposed evaluation of the effect of Lean implementation should be for multi-stage dynamic properties [1]. M.E. bayou and a.de korvin to Honda company as a benchmark, was evaluated using fuzzy logic to Ford and General Motors lean degree[2].Farzad Behrouzi and Kuan Yew Wong not only with respect to the evaluation of the importance of Lean implementation, and eliminate waste from the fuzzy theory and punctual supply to build lean assessment model, while the evaluation of the lean enterprise level [3]. In China, ErShi Qi, Wenming Cheng to import Toyota production enterprises to establish a level of nine evaluation index, evaluation was carried out [4]. Hongliang Zhang establish a lean management evaluation system, and use of the network hierarchy process and set of analysis and evaluation of the company's lean level [5].

The existing literature in the evaluation process was not taking into account the following two questions : (1) the level of lean qualitative and quantitative analysis; (2) Index selection problem. That resulting in most of the evaluation results are often significant differences between appearance and reality of the phenomenon, and corporate executives in the actual Workshop lean improvement cannot provide directional countermeasures and suggestions. In view of this, the paper creative enterprise workshop for the research background, the establishment of evaluation index system workshop lean level, and based on ANP evaluation model was constructed lean levels, at last help enterprises to provide practical advice and countermeasures in the process of lean improvement. 


\section{The lean workshop level index system}

The determination of evaluation criterion and the component index.

Lean production in essence understanding is a kind of idea, and support the idea behind is a basis: continuous improvement and two pillars: automation and just-in-time [8]. So when the index system of evaluation target as the lean workshop level, degree of automation, just-in-time and continuous improvement is the system of evaluation criteria.

In this paper, in combination of the documents on the basis of all indicators, and asked for more lean expert opinion and the suggestion, adhere to the scientific, comprehensive, objective, typical and practical principles, through reorganization, merger, cuts, refining, and ultimately build the lean level evaluation index system of the workshop. Level index system of workshop lean, Target: Automation degree A; Criterion, Workshop lean level B1, Just-in-time degree B2, Continuous improvement degree B3; Index: Equipment intelligent B11,Information B12,Inventory B21,Time B22,Pull system B23,Quality B31,Organization and personnel B32,Culture and system B33, Management B34.

\section{ANP system network structure diagram}

According to the above indicators can be constructed to build a lean level of the ANP evaluation of the structure of the shop (see the Figure 1,below)

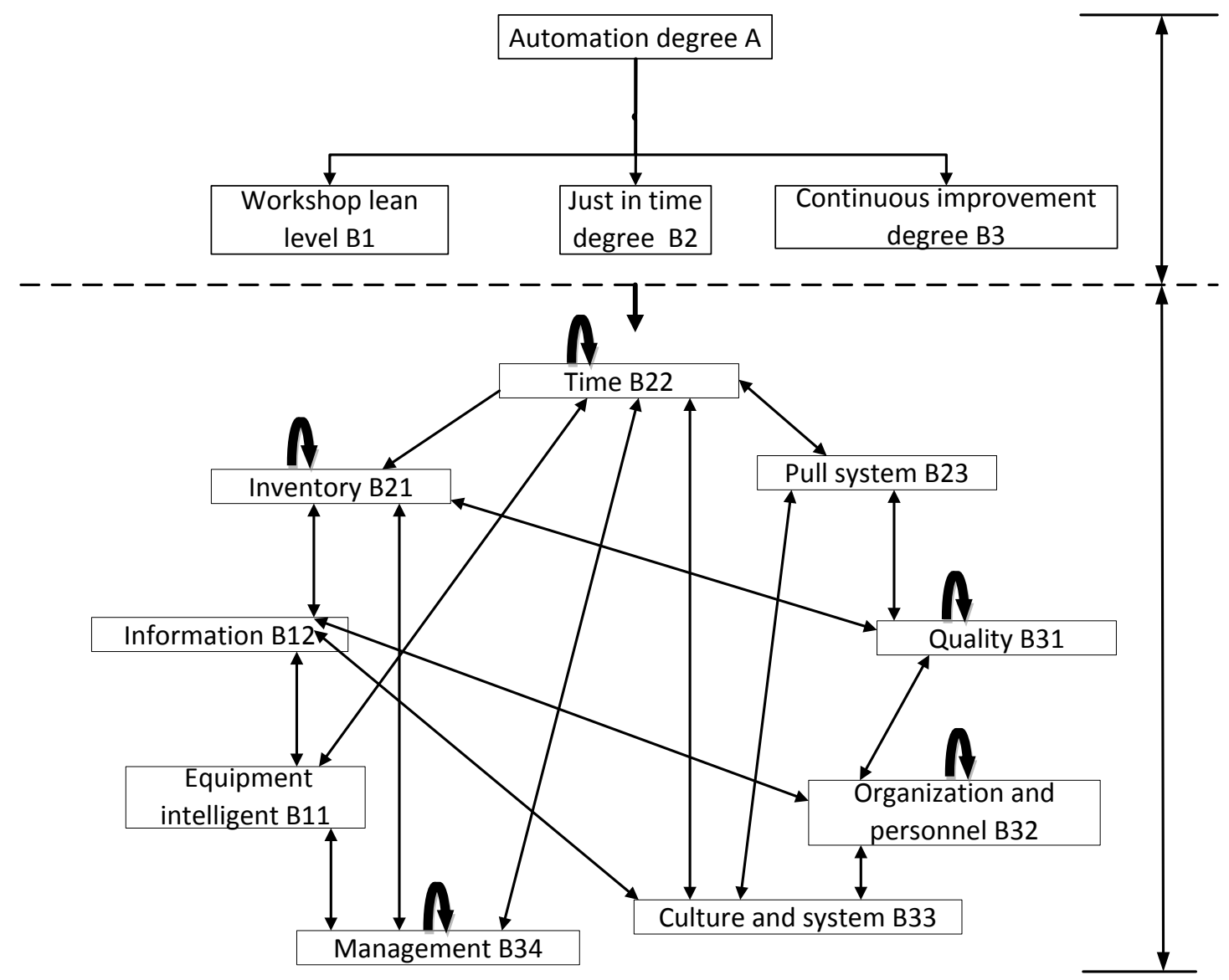

Fig. 1 Structural model of ANP evaluation for workshop lean level

\section{The calculation of the weight of each index}

According to the relationship between the elements, the evaluation value is inputted super decision software, lean workshop level of each index weight (see the Table 1, below) can be drawn. 
Table 1 The weight of each index in the workshop

\begin{tabular}{cccc}
\hline Criterion & weight & Index & weight \\
\hline Workshop lean & \multirow{2}{*}{0.29} & equipment intelligent B11 & 0.1921 \\
level B1 & & Information B12 & 0.0979 \\
Just-in-time degree & \multirow{2}{*}{0.37} & inventory B21 & 0.0723 \\
B2 & & Time B22 & 0.1886 \\
& & Pull system B23 & 0.1091 \\
Continuous & \multirow{2}{*}{0.34} & Orgality B31 & 0.1333 \\
improvement degree & \multirow{2}{*}{ Culture and system B33 } & 0.0494 \\
B3 & & management B34 & 0.0945 \\
\hline
\end{tabular}

The level of lean workshop under the combined effect of various factors, also every factors by many sub factors common role. The lean level from table 1, the workshop for: just-in-time level B2 > continuous improvement degree of B3 > level automation B1, so the workshop can strengthen the degree of automation of construction to enhance the lean level. Managers should be in the workshop of the improvement of these four aspects emphatically, for example: improve inventory turnover rate and utilization rate of the warehouse, using the training and other activities increase employees' corporate culture value and esteemed organization a certain amount of activities to promote the staff's participation rate, etc., and improve the level of E lean workshop.

\section{Summary}

Based on workshop as the background, this paper established the lean workshop level evaluation index system, and based on ANP to build the evaluation model of the lean level, at the same time with the help of a Super computer software to determine the Decision workshop of the index of the lean level achieved qualitative and quantitative analysis, the purpose is to make the enterprise have diversity to improve the workshop and a direction towards benchmarking enterprise lean level continue to improve, to strengthen the enterprise survival and development ability.

\section{References}

[1]. Sanjay Bhasin. Lean and performance measurement. Journal of Manufacturing Technology Management, Vol.19(2008), No. 5.

[2]. Fawaz A. Abdulmalek,Jayant Rajgopal. Analyzing the benefits of lean manufacturing and value stream mapping via simulation: A process sector case study. International Journal of Production Economics, Vol.107(2006), No. 1, pp.223-236.

[3]. M.E. Bayou,A. de Korvin. Measuring the leanness of manufacturing systems-A case study of Ford Motor Company and General Motors[J]. Journal of Engineering and Technology Management, Vol.25 (2008), No. 4,pp.287-304.

[4]. Ershi Qi. Civilization. TPS evaluation index system establishment and application. Science science and management of science and technology, Vol,4(2005),pp.141-143+148.

[5]. Hongliang Zhang, WenZhan Niu. Level of systematic evaluation of industrial engineering to implement lean management based on ANP-SPA, Industrial Engineering , Vol,2 (2013),pp.97-103. 